\title{
interview
}

\section{Warming up}

\author{
Room-temperature, optically pumped $\mathrm{GaAs} / \mathrm{AlGaAs} / \mathrm{GaAs}$ nanowire lasers have been realized. \\ Dhruv Saxena and Sudha Mokkapati from the Australian National University discuss this breakthrough.
}

Why work with GaAs/AIGaAs/GaAs? (Al) GaAs is the most widely studied and used III-V semiconductor system for planar optoelectronic devices. In spite of research groups having mastered the synthesis and crystal phase control of GaAs nanowires, their application has been very limited because of the very large surface recombination velocity of GaAs. For planar structures, it is possible to grow 'surfacefree' layers by taking advantage of the availability of larger bandgap, lattice-matched semiconductors. For nanowires, the surface recombination problem is accentuated because of the large surface-to-volume ratio. We tackled this issue by passivating GaAs nanowires so that they have improved properties for device applications.

How do you fabricate and optically excite the nanowires?

Our nanowires are grown by the vapourliquid-solid growth mechanism in a metal organic chemical vapour deposition (MOCVD) system. Vapour-liquid-solid growth is usually carried out at much lower temperatures than standard MOCVD growth for planar layers. We grow the nanowires on GaAs substrates using 250-nm-diameter gold nanoparticles as a catalyst. We first grow the GaAs core, which is $250 \mathrm{~nm}$ in diameter. To increase the nanowire diameter and to passivate the surface of the high-quality core, an epitaxial AlGaAs shell is grown around it at a much higher temperature. Finally, to prevent oxidation of the AlGaAs shell, an outer GaAs cap layer is grown around the shell. For optical pumping experiments, the nanowires are transferred from the growth substrate to $\mathrm{SiO}_{2}$ substrate coated with an indium-tin-oxide layer. We then use a 522-nm-wavelength laser to excite carriers above the GaAs bandgap. The spot size is large enough to excite carriers in the entire nanowire. Emitted photons are collected through the same objective used to optically excite the nanowires.

What factors are critical for lasing? High material quality, good surface passivation and a suitably designed cavity for optical feedback are all essential for achieving lasing. We use a two-temperature growth process to grow twin-defect-free GaAs cores.

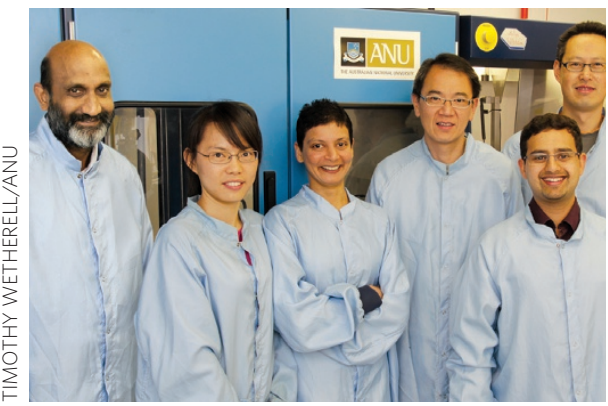

In front of the MOCVD reactor used for growing nanowires are, from left to right, Chennupati Jagadish, Nian Jiang, Sudha Mokkapati, Hark Hoe Tan, Dhruv Saxena and Qiang Gao.

We minimize surface recombination by growing a high-quality $\mathrm{AlGaAs}$ shell around the core - because of its larger bandgap, the shell prevents carriers injected into the core from reaching the surface states. Our nanowire geometry forms an intrinsic FabryPérot cavity in which light is guided along the axis of the nanowire and is reflected from its end facets. It is important for the nanowires to be untapered to ensure that modes are supported uniformly along the nanowire. In our work, we optimized the nanowire growth conditions to minimize tapering. Both the nanowire diameter and uniformity are important factors for cavity design.

Why is room-temperature lasing important, and what is the size limitation? Room-temperature operation is essential for practical devices. So far it has been unachievable for GaAs nanowires because of material quality issues. The larger bandgap $\mathrm{AlGaAs}$ shell passivates our defect-free GaAs nanowires, making lasing possible. GaAs is a technologically important material that is widely used in present optoelectronic devices. Our demonstration of room-temperature GaAs nanowire lasers opens up opportunities for smaller devices that can be integrated with three-dimensional architectures.

GaAs emits in the near infrared, which is important for short-distance optical communication. Hence, our GaAs nanowire lasers may potentially find applications in high-density data and optical communication systems. It is envisaged that nanowire lasers will be used for interand intra-chip communication via optical interconnects, and that these chips will reap faster processing speeds and lower power consumptions for future computing-based devices. The emission from the end facets of the nanowire lasers can be easily coupled to fibres and waveguides, making the nanowires suitable for lab-on-chip applications. These lasers can also be used for ultrasensitive optical sensors and scanning near-field optical microscopy.

Based on our extensive design and simulations, we found that the nanowire diameter needs to be larger than $\sim 330 \mathrm{~nm}$ to support guided modes with a low lasing threshold. The nanowire must be longer than half the excitation wavelength to support Fabry-Pérot oscillation.

What was the efficiency of your device, and will electrical pumping be possible? Our device has a slope efficiency of $\sim 50 \%$, considering the fraction of excitation power absorbed in the nanowire. For electrical pumping, the first challenge is to grow doped radial and axial heterostructures in nanowires. Selective contacts are then required for the doped regions in a single nanowire. Electrical pumping will challenge the current state-of-theart nanocharacterization and fabrication techniques. The device design will also need to be modified to account for the doping and positioning of the contact layers.

What was the biggest challenge, and what do you intend do next? Integrating a high-quantum-efficiency material and a good-quality Fabry-Pérot cavity into the nanowire growth was the main challenge. Minority carrier lifetimes of $\sim 1 \mu \mathrm{s}$ have been achieved for planar (Al)GaAs layers, whereas the minority carrier lifetime in our nanowires is only a few hundred picoseconds. We thus believe that there is still a lot of scope for improving nanowires in this material system.

\section{INTERVIEW BY DAVID PILE}

Dhruv Saxena and co-workers have a Letter describing room-temperature optically pumped GaAs/AlGaAs/GaAs nanowire lasers on page 963 of this issue. 


\section{Correction}

The print version of the Interview entitled "Warming up" (Nature Photon. 7, 1008;

2013) contained an incorrect version of the photograph, which was missing one group member listed in the figure caption. This photograph has been replaced with the correct one in both the HTML and PDF versions of the Interview. 